\title{
BMJ Open Positive and negative behaviours in workplace relationships: a scoping review protocol
}

\author{
Joan Almost, ${ }^{1}$ Angela Wolff, ${ }^{2}$ Barbara Mildon, ${ }^{3}$ Sheri Price, ${ }^{4}$ Christina Godfrey, ${ }^{1}$ \\ Sandra Robinson, ${ }^{5}$ Amanda Ross-White, ${ }^{6}$ Sheile Mercado-Mallari ${ }^{7}$
}

To cite: Almost J, Wolff A, Mildon B, et al. Positive and negative behaviours in workplace relationships: a scoping review protocol. BMJ Open 2015;5:e007685. doi:10.1136/bmjopen-2015007685

- Prepublication history for this paper is available online. To view these files please visit the journal online (http://dx.doi.org/10.1136/ bmjopen-2015-007685).

Received 15 January 2015 Accepted 20 January 2015

CrossMark

For numbered affiliations see end of article.

Correspondence to

Dr Joan Almost;

joan.almost@queensu.ca

\section{ABSTRACT}

Introduction: Engaging in teamwork requires a clear understanding of positive and negative behaviours that act as facilitators and barriers to collegial workplace relationships. Identifying and correcting underlying barriers, while promoting facilitators, is fundamental to improving care delivery and, ultimately, clinical outcomes. Despite a considerable amount of literature in this area, there is a lack of clarity of the different behaviours as several parallel literatures address similar questions about antecedents, processes and outcomes. The purpose of this study is to synthesise the current state of literature reporting on behaviours in workplace relationships. Using a scoping review methodology, the following research question will be addressed: "What is known about positive and negative behaviours in workplace relationships?"

Methods and analysis: We will employ the methodological frameworks used by Arksey and O'Malley and Levac et al. The search strategy will include numerous electronic databases, grey literature sources and hand-searching of reference lists from 1990 to present with a limit to English language. Search strategies will be developed using controlled vocabulary and keyword terms related to various components of workplace relationships. Two reviewers will independently screen titles and abstracts for inclusion, followed by screening of the full text of potential articles to determine final inclusion. A descriptive numerical analysis will describe characteristics of included studies. A thematic analysis will provide an overview of the literature, including definitions, conceptual frameworks, antecedents, outcomes and interventions.

Dissemination: In reviewing a wide range of positive and negative behaviours, then integrating into a manageable, meaningful whole, this study is a critical step in helping policymakers, leaders and healthcare professionals effectively use what is known thus far. Knowledge translation activities will occur throughout the study with dissemination of findings to local, national, and international stakeholders, including a wide range of clinicians, leaders and administrators in all sectors.

\section{INTRODUCTION}

Quality patient-centred care requires collegial workplace partnerships among healthcare

\section{Strengths and limitations of this study}

- Supports a partnership between researchers and point-of-care knowledge users throughout the enquiry, from conceptualisation to knowledge dissemination.

- Strengthens the breadth of analysis by tapping into literature on positive and negative behaviours from across disciplines and sectors.

- Guided by a validated methodological framework and systematic approach.

- This review will be limited to the English language only.

- The quality of the evidence will not be evaluated (as this is a scoping review).

team members. A prerequisite for such partnerships includes respectful, collaborative working relationships. Collaborative workplace relationships are consistently linked to positive outcomes and often compensate for other difficulties that may arise in the workplace. ${ }^{1-3}$ Effective teamwork and communication have been cited as reducing stress and burnout, ${ }^{4} 5$ increasing job satisfaction, recruitment and retention, improving patient satisfaction and reducing patient mortality. ${ }^{12}$ Conversely, ineffective workplace relationships result in impaired teamwork, communication and decision-making and are associated with poorer work performance, greater absenteeism, professional disengagement and more rapid job turnover. ${ }^{6-9}$ Within healthcare, it is well known that communication failures and ineffective teamwork can lead to patient safety incidents and patient harm. ${ }^{4510}$

Although the value of teamwork is well recognised in healthcare, the dynamics of workplace relationships creates a myriad of challenges for healthcare teams. In today's increasingly complex and stress-laden healthcare environments, relationships can be threatened by negative behaviours such as conflict, emotional abuse, bullying and incivility. Negative behaviour is a problem within and 
across all healthcare professional groups, however, the international literature suggests that negative behaviour, such as conflict, bullying, harassment and horizontal violence, is the greatest problem intraprofessionally within nursing, in its prevalence and level of distress it causes. ${ }^{11-15}$ Interpersonal workplace relationships are an important component of working life and poor relations with colleagues strikes at the heart of why so many frontline nurses are stressed, disheartened and on the verge of burning out. ${ }^{12}$ A wealth of research demonstrates that the quality of work environments, including positive interpersonal relationships, impacts not only organisational measures, but also patient outcomes. ${ }^{16-19}$ Knowledge gaps related to optimising teamwork within healthcare hinders the development of targeted, effective, quality enhancement and patient safety initiatives. Therefore, engaging in teamwork requires a clear understanding and articulation of the positive and negative behaviours that act as facilitators and barriers to effective collegial workplace relationships. Identifying and correcting these underlying barriers, while promoting facilitators, is fundamental to a sound strategy to improve care delivery and, ultimately, clinical outcomes.

International scholars and practitioners from many disciplines have shown great interest in understanding the various behaviours of workplace relationships. ${ }^{20} \quad 21$ The literature identifies a wide range of positive and negative behaviours. Collegiality, respect, cooperation, teamwork, social support, mentorship and collaboration are the terms often used to describe positive relationships among colleagues. Negative relationships have included terms such as horizontal violence, aggression, bullying, verbal abuse, incivility, lateral violence, ostracism, interpersonal conflict, negative acts and disruptive behaviour. Overarching constructs such as workplace aggression, ${ }^{22}$ psychological aggression, ${ }^{23}$ negative behaviour, ${ }^{24}$ and negative relationships ${ }^{25}$ encompass physical violence, harassment, bullying, emotional abuse, abusive supervision and workplace incivility. Theoretical reviews have focused mostly on the negative behaviours of workplace relationships with attempts to integrate the diverse literatures. ${ }^{26-30}$ However, most of this work has taken place in non-healthcare settings and have not included an integration of the positive behaviours.

Despite the considerable amount of literature in this area, there continues to be a lack of standardised definitions and conceptual clarity of the different behaviours. ${ }^{31} 32$ This lack of agreement on concepts and definitions has impeded the building of a relevant literature base and conceptual framework on collegial workplace relationships. We will build on the previous reviews to determine the applicability of the findings to healthcare settings and include an integration of positive behaviours in addition to the negative behaviours. The strength of this review is the breadth of analysis that will be enabled by tapping into international literature from across disciplines and sectors. In reviewing a wide range of different construct labels, measures and findings, then seeking to integrate and summarise the constructs into a manageable, meaningful whole we hope to identify the primary causal antecedents and interventions known to reduce negative workplace behaviours and increase positive behaviours. This integration and simplification is a critically necessary task if we hope for policymakers, healthcare leaders and healthcare professionals to effectively use what we know thus far, enabling them to more effectively manage and reduce corrosive behaviour and increase collaborative behaviour in healthcare settings.

In this scoping review, we propose to review and clarify the 'state of knowledge' from the literature reporting on behaviours of workplace relationships. We will address this with the engagement of knowledge users in leadership, policy and practice to design, analyse and disseminate evidence to address the following objectives: (1) synthesise existing evidence through a comprehensive review of published and grey literature on the key concepts related to workplace relationships; (2) identify and summarise the types of positive and negative behaviours of workplace relationships being conceptualised; (3) create a conceptual framework of positive and negative behaviours, which includes antecedents, outcomes, definitions, characteristics and supporting underlying theories; (4) generate a list of instruments used to measure concepts identified in conceptual framework; and, (5) generate a preliminary list of interventions shown to promote collegial workplace relationships with recommendations for future research priorities based on knowledge gaps for interventional research.

\section{METHODS AND ANALYSIS}

Scoping reviews are exploratory studies that systematically map the literature on a selected topic. ${ }^{33}$ The four major functions include mapping the current state of literature, determining feasibility of conducting a systematic review, summarising and disseminating research findings to knowledge users, and identifying gaps where further research is required. ${ }^{34}{ }^{35}$ Scoping reviews share several characteristics of the systematic review including being systematic, transparent and replicable. ${ }^{36}$ Utilising an integrated-knowledge translation approach, Arksey and O'Malley ${ }^{34}$ and Levac et $a l^{35}$ developed a six-stage methodological framework: (1) identification of the research question(s) to be addressed; (2) identification of studies relevant to research question(s); (3) selection of studies to include in the review; (4) charting of information and data within the included studies; (5) collating, summarising and reporting results of the review; and (6) consultation with stakeholders to ensure comprehensive inclusion of all relevant material. This approach will guide this synthesis.

\section{Stage 1: Identification of research question to be addressed}

In scoping reviews, research questions should be broad as the focus is on summarising the breadth of evidence. ${ }^{34}$ Linking a clear purpose to a well-defined 
research question at the first stage provides a strong rationale for completing the study and facilitates decision-making about study selection and data extraction later in the process. ${ }^{35}$ An overall research question with three subcomponents will guide the selection of relevant sources: What is known about the positive and negative behaviours in workplace relationships?

1. What are the antecedents, outcomes, definitions, characteristics and conceptual frameworks of the behaviours?

2. What instruments are used to measure each of the behaviours?

3. What interventions have been effective in improving workplace relationships?

The national collaboration with knowledge-users will be twofold: to provide the essential point-of-care, educational, and policy perspectives, and, to develop the additional knowledge translation tools that will be required to assist practitioners in the uptake of the synthesis results. The focus area and research questions for this study were developed together by the knowledge users and researchers early in the development of this protocol. The knowledge users stressed the importance of extrapolating promising tools and interventions as practical outputs. In order to ensure the rigour, comprehensiveness, and relevance of this review, an expert panel of 10-15 individuals from across Canada will be assembled including content experts, policymakers, and professional groups. In the first of two interactive meetings, the expert panel will be asked to participate with the research team in a web conference to refine the purpose and context of this review. This early involvement will engage the healthcare community in the development of the study outcomes, create awareness of the study and provide opportunities for knowledge exchange.

\section{Stage 2: Identification of studies relevant to the research question}

This stage involves identifying relevant studies and developing a search strategy, terms to use, sources to be searched, time span and language. ${ }^{34}$ The search strategy will involve a range of approaches and will be developed further with the library scientist on the research team to locate published and unpublished literature. To obtain a breadth of conceptual understanding and to support the exploratory nature of the review, the search strategy will not be limited to nursing or healthcare settings. The review will include: (1) published or unpublished primary studies, theses/dissertations, theoretical discussions and grey literature; (2) all levels of analyses (unit, group, organisation); and, (3) all levels of employees. All literature database searches will be searched from 1990 to present and limited to the English language. Given our knowledge of the literature, this time period was chosen to capture many key articles published in the 1990s. The research team will meet to discuss decisions surrounding study inclusion and exclusion at the beginning of the process. With increasing familiarity of the literature, the research team will refine criteria to determine the relevance of the citations.

To be comprehensive, multiple literature sources will be used, including electronic databases and reference lists of relevant literature. Electronic searching will result in lists of articles with details of title, author/s, source and possibly abstracts. We will search the following electronic databases: Cumulative Index to Nursing and Allied Health Literature (CINAHL; 1982 to present); MEDLINE (1950 to present); EMBASE (1947 to present); Web of Science (including Science Citation Index (1945 to present) and Social Sciences Citation Index (1956 to present) and Arts and Humanities Citation index (1975 to present); PsycINFO (1967 to present); Sociological Abstracts; ISI Proceedings (including Conference Proceedings Citation IndexScience (1990 to present), Conference Proceedings Citation Index-Social Science and Humanities (1990 to present), Cochrane Effective Practice and Organisation of Care Group's Trials Register (2012); Cochrane Central Register of Controlled Trials; Current Contents (1994 to present), Joanna Briggs and ProQuest Dissertations and Theses.

Search terms will include controlled vocabulary and keyword terms related to (1) the various components of workplace relationships AND (2) terms for workplace. The search terms will be applied according to the nuances of each database. Keywords will include, but not be limited to (1) conflict (intragroup or interpersonal), bullying, incivility, violence, aggression, harassment, dysfunctional, negative relationships, negative acts, negative behaviour, abuse, horizontal violence, lateral violence, ostracism, social undermining, respect, collegiality collaboration, communication, civility, cooperation, collaborative, teamwork, partnership, mentorship, and (2) work environments, work relationships, interpersonal, workplace relations, work conditions, workplace, intragroup. A preliminary search was conducted by the library scientist using the following PICO question: What is known about the positive and negative components of workplace relationships. Two databases were searched: CINAHL and PsycINFO. The CINAHL search strategy produced 2980 while the PsycINFO search produced 3087 results.

Once relevant material is selected from the electronic databases, reference lists of all studies will be reviewed to ensure all relevant material is captured. Several websites will also be targeted for unpublished literature such as the Agency for Healthcare Quality and Research (http://www. ahrq.gov/), American Nurses Association (http://www. nursingworld.org/), Academy of Management (http:// www.aomonline.org/) and other sites identified by the research team and expert panel.

\section{Stage 3: Selection of studies to include in the review}

Refining the search strategy based on abstracts retrieved from the search and reviewing full articles for study inclusion is a critical step. ${ }^{35}$ Owing to the large number of anticipated abstracts, two graduate students will 
independently screen the titles and abstracts yielded from the search strategy for study selection. This will help to ensure that the abstracts selected are relevant for full article review. When in doubt about the relevance of a publication, the full text will be retrieved to determine its relevance. To ensure the selection process is non-biased, two team members will be asked to review 50 abstracts independently and their results will be compared with papers selected by the initial panel. At this stage, the research question may require refinement to ensure the review is feasible, without compromising the comprehensiveness of the search. Next, the graduate students will independently review the full articles for inclusion. Disagreements will be resolved through consensus and if consensus cannot be reached, the research team leads will be consulted to determine final decision. A screening tool will be developed by the research team and expert panel to determine relevance of papers to workplace relationships and healthcare settings and code the type of data provided (eg, empirical data, reviews and theoretical discussions). All graduate students will have received training from the Queen's Joanna Briggs Collaboration. The research team will meet on a monthly basis during this stage to discuss challenges and uncertainties related to study selection. Search strategies and/or inclusion criteria will be further refined if needed. A unique identifier will be assigned to every publication retrieved in the search process. This will enable tracking of articles throughout the review process. RefWorks will be used to manage the results of all searches and to facilitate the screening process with each reviewer documenting the inclusion/ exclusion status and rationale for each study.

\section{Stage 4: Charting of information and data within the included studies}

A 'descriptive analytical' method will be used to extract contextual or process oriented information from each study. ${ }^{34}$ This technique includes synthesising and interpreting data by sifting, charting and sorting material according to key issues and themes. The study team will collectively develop a data-charting form to determine which variables to extract that will help to answer the research questions. This charting is considered an iterative process in which the form will be continually updated. ${ }^{35}$ The research team leads and graduate students will independently extract data from the first $5-10$ studies using a data-charting form and meet to determine whether their approach to data extraction is consistent with the research questions and purpose. Following this, the graduate students will independently extract data from each included publication. The research team leads will double check the extracted data for accuracy and completeness. Studies will be categorised by the different components, such as conflict, bullying, incivility, collaboration, and so forth, as well as by type of study design (quantitative, qualitative, discussion papers), and healthcare settings versus non- healthcare settings. Extracted data will include: year, authors, publication title, research question or study purpose, study design, context, participants, sample size, theoretical/conceptual framework, intervention (type, elements), definitions of concepts, data collection methods, instrumentation and relevant results.

\section{Stage 5: Collating, summarising and reporting results of the review synthesis}

The stages of a scoping review are similar to those of a systematic review, however, due to the vast and diverse literature, scoping reviews do not include a detailed appraisal of identified evidence sources. Instead scoping reviews collate the evidence using a descriptive numerical summary and a thematic analysis. ${ }^{34}$ The descriptive numerical analysis will be done by two graduate students to describe the characteristics of included studies, such as the overall number of studies, types of study design, years of publication, types of interventions, characteristics of the study populations and countries where studies were conducted. The second summary will be a thematic analysis to provide an overview of the breadth of the literature. ${ }^{34}$ Potential themes that emerge from the review will be compiled into a topic matrix to allow easy comparison by topic. For each of the concepts, we will produce tables and charts mapping: (1) the antecedents, outcomes, definitions, characteristics and supporting underlying theories; (2) the instruments used to measure each of the concepts, including psychometric properties; and (3) the effectiveness of interventions that were found in the review. The key concepts of positive and negative behaviours will be the primary unit of analysis unless it is determined that certain concepts can be combined. By applying a consistent approach to reporting the findings we will be able to make comparisons across concepts; identify similarities and differences, develop a conceptual framework, and identify gaps. The initial thematic analysis will be completed by the research team leads and two graduate students. The analysis will then be shared with the research team and reviewed in a face-to-face team meeting. During this 2-day meeting, the research team will also consider the best approach to stating the outcome or end product of the study and how the scoping review findings will be articulated to the expert panel in the next meeting.

\section{Stage 6: Consultation with stakeholders}

The final consultation stage offers an ideal mechanism to enhance the validity of the study outcome while translating findings with the healthcare community. Preliminary findings from the review will provide the foundation for a $4 \mathrm{~h}$ interactive web conference with the research team and expert panel. The objectives of the web conference are to present and discuss the interim results of the synthesis. The meeting will generate a list of key practice recommendations, dissemination strategy and research priority areas to inform future research. A final $3 \mathrm{~h}$ web conference will be conducted with the 
research team with a focus on reviewing the final results following feedback from the previous meeting, a review of the final report, necessary modifications to findings and recommendations for precision and clarity. Particular attention will be paid to strategic planning for dissemination of the recommendations by members such as formal and informal presentations in practice settings, websites and newsletters.

\section{ETHICS AND DISSEMINATION}

The aim of this project is to synthesise the literature from a broad range of disciplines about workplace relationships in order to enhance conceptual clarity and understanding about key barriers and facilitators to collegial workplaces and to extrapolate from this knowledge base promising tools and interventions that may be relevant for the health sector in particular. We anticipate the research will provide several key outputs including (1) a comprehensive review that will summarise and integrate existing literature; (2) an integrated conceptual framework; (3) a list of standardised instruments; and, (4) a preliminary list of interventions that could be implemented and evaluated in practice settings. Our goal will be to disseminate our findings to a wide range of clinicians, leaders and administrators in all sectors, to researchers and to students entering the healthcare professions to enhance understanding about key facilitators and barriers of healthy workplaces and to seed further research about promising interventions for healthcare settings.

A technical report of the full enquiry will be created outlining the scope, findings and recommendations. Context specific journals will be targeted for publications given the high relevance of our findings to their foci. The research findings will also be reported in a succinct, non-technical paper discussing the relevance of the findings for health professionals, senior administrators and policymakers, the application of the findings to practice and the recommendations. A series of two-page summary sheets will be created for clinicians. The technical report will be made available through the websites of the research team members and expert panel. The non-technical report and summary sheets will also be posted on the websites of professional organisations. In addition, reports and summary sheets will be disseminated through the existing networks of the research team, knowledge users and expert panel from various clinical groups, healthcare authorities, professional associations, to specific audiences (eg, administrators, researchers, educators, health professionals).

A webinar will be developed discussing the study, its results and recommendations. The Canadian Nurses Association (CNA) has committed support to assisting with dissemination of the study's findings including development of a webinar through their NurseOne network, and posting information about the study and results on their website and through CNA publications. The existence of the webinar will be announced through our knowledge users, expert panel, CNA's publications, at conferences, healthcare practitioner listservs and published material from the study. Presentations and/or poster presentations at national, international conferences and local conferences will be organised to reach a wide audience of practitioners. Presentations and interactive workshops will also be made to front-line healthcare professionals from each sector at the local level by reaching out to contacts within each of the regions represented on the research team (Ontario, Nova Scotia, British Columbia). A popular and typically well-attended venue is preconference workshops. A half-day preconference seminar will be developed and delivered at a national conference. At each of these seminars, team members will present the research study and host an interactive session to discuss the results and implications for practice and policy. These sessions in themselves will assist us in gaining further understanding from participants as to the applicability of the results and how to further enhance knowledge translation. This would be a highly feasible avenue for the transfer of this knowledge. Members of the research team have previous experience at local, national and international conferences conducting workshops. The results will also be disseminated through interactive education seminars with practitioners and students at the workplace of each of the research team members. The interactive seminars will discuss the study, its findings, implications, barriers to the implementation of the evidence, and strategies to overcome these barriers. Lastly, we believe the upcoming generation of practitioners needs to be included in the dissemination strategy. Presentations will be tailored to healthcare students at Queens University, Dalhousie University, and the University of British Columbia. These will be delivered in interdisciplinary groups to encourage discussion and participation. Students are well positioned to adopt new knowledge as they enter the practice arena.

This study is a critical step in helping to inform interventions to foster collegial and respectful relationships as point-of-care healthcare professionals strive to provide quality patient care. This study will address a gap in the literature with the inclusion of positive behaviours as well as negative behaviours. These findings will assist health system decision-makers in identifying standardised and comprehensive definitions and conceptual frameworks of positive and negative workplace behaviours. As part of our scoping review, we will generate a preliminary list of interventions that have been shown to promote collegial workplace relationships. This tangible product will provide very practical information for use by knowledge users to inform education programme development and interventions in the workplace for dealing with issues and challenges regarding workplace behaviours, and in shaping policies. Ultimately this review will provide knowledge users with the information they need to make evidence-informed decisions to optimise and enhance effective teamwork and, ultimately, improve patient care. 
Author affiliations

${ }^{1}$ School of Nursing, Queen's University, Kingston, Ontario, Canada

${ }^{2}$ Department of Clinical Education, Professional Practice and Integration,

Fraser Health Authority, Surrey, British Columbia, Canada

${ }^{3}$ Professional Practice, Human Resources, Research and Chief Nurse

Executive, Ontario Shores Centre for Mental Health Sciences, Oshawa, Ontario, Canada

${ }^{4}$ School of Nursing, Dalhousie University, Halifax, Nova Scotia, Canada

${ }^{5}$ Sauder School of Business, University of British Columbia, Vancouver, British Columbia, Canada

${ }^{6}$ Bracken Health Sciences Library, Queen's University, Kingston, Ontario, Canada

${ }^{7}$ Workplace Health, Fraser Health Authority, Surrey, British Columbia, Canada

Contributors JA was involved in study conception, writing of the protocol, preliminary literature review, content expert input (research, reviews), scoping review frameworks, analysis; AW was involved in study conception, editing of protocol, content expert input, stakeholder input; BM was involved in stakeholder input; SP was involved in editing of protocol, content expert input; CG was involved in editing of protocol, content expert input; SR was involved in editing of protocol, content expert input; AR-W was involved in editing of protocol, search strategy; SM-M was involved in stakeholder input.

Funding This work was supported by a Canadian Institutes of Health Research Knowledge Synthesis Grant \#126598 and co-sponsored by the Canadian Nurses Association, Fraser Health Authority, and Queen's Joanna Briggs Collaboration.

Competing interests None.

Provenance and peer review Not commissioned; internally peer reviewed.

Open Access This is an Open Access article distributed in accordance with the Creative Commons Attribution Non Commercial (CC BY-NC 4.0) license, which permits others to distribute, remix, adapt, build upon this work noncommercially, and license their derivative works on different terms, provided the original work is properly cited and the use is non-commercial. See: http:// creativecommons.org/licenses/by-nc/4.0/

\section{REFERENCES}

1. Borrill C, West MA, Shapiro D, et al. Team working and effectiveness in health care. $\mathrm{Br} J$ Health Care Manag 2000;6:364-71.

2. D'Amour D, Ferrada-Videla M, San Martin Rodriguez L, et al. The conceptual basis for interprofessional collaboration: core concepts and theoretical frameworks. $J$ Interprof Care 2005;19:116-31.

3. Evans M. On the job. Nurses reveal most, least enjoyable aspects of work. Mod Healthc 2005;35:14-15.

4. Canadian Institute for Health Information. Canada's health care providers. Ottawa, ON: Canadian Institute for Health Information, 2007. https://secure.cihi.ca/free_products/HCProviders_07_EN_final. pdf (accessed 7 Mar 2012)

5. Lomas J, Culyer T, McCutcheon C, et al. Conceptualizing and combining evidence for health system guidance: final report. Ottawa, ON: Canadian Health Services Research Foundation, 2005. http:// www.cfhi-fcass.ca/migrated/pdf/insightAction/evidence_e.pdf (accessed 3 Sep 2012).

6. Hughes A. Being bullied what an insight. Br J Perioper Nurs 2003;13:166-8.

7. Farrell GA, Bobrowski C, Bobrowski P. Scoping workplace aggression in nursing: findings from an Australian study. J Adv Nurs 2006;55:778-87.

8. Sofield L, Salmond SW. Workplace violence. A focus on verbal abuse and intent to leave the organization. Orthop Nurs 2003;22:274-83.

9. Stevens $S$. Nursing workforce retention: challenging a bullying culture. Health Aff 2002;21:189-93.

10. Sutcliffe KM, Lewton E, Rosenthal MM. Communication failures: an insidious contributor to medical mishaps. Acad Med 2004;79:186-94.

11. Almost J, Doran D, McGillis-Hall L, et al. Antecedents and consequences of intragroup conflict among nurses. J Nurs Manag 2010;18:981-92.
12. Canadian Health Services Research Foundation. What's ailing our nurses? A discussion of the major issues affecting nursing human resources in Canada. Ottawa, ON: Canadian Health Services Research Foundation, 2006. http://www.cfhi-fcass.ca/Migrated/PDF/ What sailingourNurses-e.pdf (accessed 3 Sep 2012).

13. Lambert VA, Lambert CE, Ito M. Workplace stressors, ways of coping and demographic characteristics as predictors of physical and mental health of Japanese hospital nurses. Int J Nurs Stud 2004;41:85-97.

14. McKenna BG, Smith NA, Poole SJ, et al. Horizontal violence: experiences of registered nurses in their first year of practice. $J$ Adv Nurs 2003;42:90-6.

15. Woelfle CY, McCaffrey R. Nurse on nurse. Nurs Forum 2007;41:123-31.

16. Laschinger HKS, Leiter MP. The impact of nursing work environments on patient safety outcomes: the mediating role of burnout engagement. J Nurs Adm 2006;36:259-67.

17. Armstrong $\mathrm{K}$, Laschinger $\mathrm{H}$, Wong $\mathrm{C}$. Workplace empowerment and magnet hospital characteristics as predictors of patient safety climate. J Nurs Care Qual 2009;24:55-62.

18. Squires M, Tourangeau A, Spence Laschinger HK, et al. The link between leadership and safety outcomes in hospitals. J Nurs Manag 2010;18:914-25.

19. Aiken LH, Cimiotti JP, Sloane DM, et al. Effects of nurse staffing and nurse education on patient deaths in hospitals with different nurse work environments. Med Care 2011;49:1047-53.

20. Monks CP, Smith PK, Naylor P, et al. Bullying in different contexts: commonalities, differences and the role of theory. Aggress Violent Behav 2009;14:146-56.

21. Keashly L, Jagatic K. North American perspectives on hostile behaviors and bullying at work. In: Einarsen $\mathrm{S}$, Hoel H, Zapf D, et al. eds. Bullying and harassment in the workplace: developments in theory, research, and practice. 2nd edn. Boca Raton, FL: CRC Press, 2011:41-74.

22. Neuman JH, Baron RA. Workplace violence and workplace aggression: evidence concerning specific forms, potential causes, and preferred targets. J Manag 1998;24:391-419.

23. Bowie V, Fisher B, Cooper CL. Introduction: new issues, trends and strategies in workplace violence. In: Bowie V, Fisher BS, Cooper C. eds. Workplace violence. London, UK: Willan Publishing, 2005:1-12.

24. Hutton SA. Workplace incivility: state of the science. J Nurs Adm 2006;36:2-27.

25. Morrison RL. Negative relationships in the workplace: associations with organisational commitment, cohesion, job satisfaction and intention to turnover. J Manag Organ 2008;14:330-44.

26. Hershcovis MS, Turner N, Barling J, et al. Predicting workplace aggression: a meta-analysis. J Appl Psychol 2007;91:228-38.

27. O'Leary-Kelly AM, Griffin RW, Glew DJ. Organization-motivated aggression: a research framework. Acad Manage Rev 1996;21:225-53.

28. Robinson SL, Bennett RJ. A typology of deviant workplace behaviors: a multidimensional scaling study. Acad Manage $J$ 1995;38:555-72.

29. Spector PE, Fox S. The stressor-emotion model of counterproductive work behavior. In: Spector PE, Fox S. eds. Counterproductive work behavior: investigations of actors and targets. Washington DC: APA Press, 2005:151-74.

30. Vessey JA, Demarco R, DiFazio R. Bullying, harassment, and horizontal violence in the nursing workforce: the state of the science. Annu Rev Nurs Res 2010;28:133-57.

31. Broome BA. Dealing with sharks and bullies in the workplace. ABNF J 2008:19:28-30.

32. Wiskow C. Framework guidelines for addressing workplace violence in the health sector: the training manual. http://www.ilo.org/safework/ info/instr/WCMS_108542/lang--en/index.htm (accessed 1 Sep 2012).

33. Canadian Institutes of Health Research 2010. A Guide to Knowledge Synthesis. http://www.cihr-irsc.gc.ca/e/41382.html (accessed 15 Jun 2012).

34. Arksey H, O'Malley L. Scoping studies: towards a methodological framework. Int J Soc Res Methodol 2005;8:19-32.

35. Levac D, Colquhoun H, O'Brien KK. Scoping studies: advancing the methodology. Implement Sci 2010;5:69.

36. Grant MJ, Booth A. A typology of reviews: an analysis of 14 review types and associated methodologies. Health Info Libr J 2009;26:91-108. 